\title{
Clinician Capsules for CJEM 20(3)
}

\author{
Mobility assessments of geriatric emergency \\ department patients: A systematic review
}

Debra Eagles, Krishan Yadav, Jeffrey J. Perry, Marie Josée Sirois, Marcel Emond

doi:10.1017/cem.2017.46

\begin{abstract}
What is known about the topic?
Multiple guidelines advise applying specific mobility assessment instruments in older ED patients.

What did this study ask?

This study sought to examine the literature on mobility assessments in older ED patients and determine if these measures were associated with adverse outcomes.

What did this study find?

Timed Up and Go was the most commonly evaluated mobility assessment in older ED patients. Mobility assessments did not predict future falls or return ED visits/hospitalization.

Why does this study matter to clinicians?

While structured and unstructured evaluation of mobility is recommended and carried out in older ED patients prior to discharge, current research does not support use of any specific measure as a predictor of future adverse outcomes.
\end{abstract}

Discrepancy between information provided and information required by emergency physicians for long-term care patients

Richa Parashar, Shelley McLeod, Don Melady

doi:10.1017/cem.2017.353

What is known about the topic?

The information accompanying long term care (LTC) residents to the emergency department (ED) is often incomplete.

What did this study ask?

What information is currently included in LTC transfer documentation and how does that compare to the information required by local emergency physicians to provide optimal care and make decisions for LTC patients?

What did this study find?

Our study demonstrates a clear discrepancy between information provided and the greater information needs required by emergency physicians for LTC patients.

Why does this study matter to clinicians?

Quality improvement initiatives at the local level may help to reduce this discrepancy.

(c) Canadian Association of Emergency Physicians

CJEM 2018;20(3):315-317

CAEP | ACMU
Between- and within-site variation in medication choices and adverse events during procedural sedation for electrical cardioversion of atrial fibrillation and flutter

David Clinkard, Ian G. Stiell, Eddy Lang, Stuart Rose, Catherine M. Clement, Robert Brison, Brian H. Rowe, Bjug Borgundvaag, Trevor Langhan, Kirk Magee, Rob Stenstrom, Jeffery J. Perry, David Birnie, George A. Wells, Andrew D. McRae

\section{doi:10.1017/cem.2017.20}

\section{What is known about the topic?}

Procedural sedation for cardioversion is a common ED practice, though limited research exists on optimal drug choice and frequency of adverse events.

What did this study ask?

What are the regional practice variations and common adverse events associated with procedural sedation for cardioversion?

What did this study find?

There was substantial variation between sites, though over time the use of propofol and fentanyl was most popular. The most common adverse event was hypoxia, and this was most strongly associated with the use of the combinations of midazolam, fentanyl and propofol.

Why does this study matter to clinicians?

The use of multiple drugs for sedation is associated with increased adverse events.

Predictors of obtaining follow-up care in the province of Ontario, Canada, following a new diagnosis of atrial fibrillation, heart failure, and hypertension in the emergency department

Clare L. Atzema, Bing Yu, Noah M. Ivers, Paula A. Rochon, Douglas S. Lee, Michael J. Schull, Peter C. Austin

doi:10.1017/cem.2017.371

What is known about the topic?

Patients with ambulatory-sensitive cardiovascular conditions are seen frequently in emergency departments (EDs), and many are discharged home at the end of the visit.

What did this study ask?

What are the independent predictors of being seen within a week of discharge by a family physician or specialist following an ED visit for atrial fibrillation, heart failure, or hypertension? What did this study find?

Half of patients were seen within a week in Ontario: lack of a family physician decreased the rate the most, while family 
physician remuneration method and patient comorbidities also played a role.

Why does this study matter to clinicians?

Emergency physicians advise these patients to obtain follow-up within a week, but they should be aware that this is happening in only half of cases, as well as the factors that reduce that likelihood.

Implementation of an emergency department atrial fibrillation and flutter pathway improves rates of appropriate anticoagulation, reduces length of stay and thirty-day revisit rates for congestive heart failure

David Barbic, Chris DeWitt, Devin Harris, Robert Stenstrom, Eric Grafstein, Crane Wu, Cristian Vadeanu, Brett Heilbron, Jenelle Haaf, Stanley Tung, Dan Kalla, Julian Marsden, Jim Christenson, Frank X. Scheuermeyer

doi:10.1017/cem.2017.418

\section{What is known about the topic?}

ED physicians frequently miss opportunities to prescribe oral anticoagulants (OACs) to patients with atrial fibrillation/flutter (AFF).

What did this study ask?

Did implementing a new evidence-based pathway improve OAC prescribing behavior?

What did this study find?

This pre-post study found a $20 \%$ absolute increase in appropriate OAC prescribing along with decreased lengths of stay and decreased return visits for congestive heart failure. Why does this study matter to clinicians?

Local implementation of a similar pathway could lead to better and more efficient care of AFF patients.

\section{Frequent users of the pediatric emergency department}

Jade Seguin, Esli Osmanlliu, Xun Zhang, Virginie Clavel, Harley Eisman, Robert Rodrigues, Maryam Oskoui

\section{doi:10.1017/cem.2017.15}

\section{What is known about the topic?}

Emergency Department Crowding is associated with increased morbidity and mortality.

What did this study ask?

What are the diagnoses and patient's characteristics associated with frequent ED use in children?

What did this study find?

Asthma, ear, nose, sinus and respiratory infections account for the largest proportion of diagnoses for which children frequently use the ED.

Why does this study matter to clinicians?

The identified risk factors and diagnoses highlight the need for future innovative and targeted strategies to minimize ED crowding.
Parental experiences and preferences as participants in pediatric research conducted in the emergency department

Antonia S. Stang, Stephen B. Freedman, Angelo Mikrogianakis, Graham C. Thompson, Janie Williamson, David W. Johnson

\section{doi:10.1017/cem.2017.22}

\section{What is known about the topic?}

Understanding research participant preferences is particularly important in children due to the necessary involvement of parents.

\section{What did this study ask?}

What are parental experiences and preferences regarding the conduct of pediatric research in an emergency department setting?

What did this study find?

Parents prefer to be approached in an area that is separate from the waiting room, and email and telephone follow-ups are preferred over a scheduled re-visit.

Why does this study matter to clinicians?

These findings will enable researchers to align the conduct and design of research in an ED setting with parental preferences.

\section{Determining the clinical significance of errors in pediatric radiograph interpretation between emergency physicians and radiologists}

Jonathan Taves, Steve Skitch, Rahim Valani doi:10.1017/cem.2017.34

\section{What is known about the topic?}

ED physicians routinely review and interpret plain radiographs in order to make clinical decisions.

What did this study ask?

What is the rate of discrepancy between an ED physician and radiologist for pediatric plain radiographs, and what is the clinical significance of those that are not interpreted correctly?

What did this study find?

This study found a $1.16 \%$ rate of discrepancy between ED physician and radiologist interpretation. Of the $>25,000$ plain radiographs completed, only $0.4 \%$ had an incorrect interpretation requiring change in management.

Why does this study matter to clinicians?

ED physicians do well in reporting pediatric plain radiographs. Further education in areas where there were discrepancies can help improve clinical practice. 
Dental injuries in younger emergency department patients

Vigil James, Yona R. Vandersluis, Evangeline W. J. Zhang, Dennis Scolnik

doi:10.1017/cem.2017.52

What is known about the topic?

Dental trauma is an unfortunate and relatively common occurrence in childhood.

What did this study ask?

The aim of this study was to examine changing patterns in the incidence and aetiology of dental injuries in Canadian patients between 0-18 years of age.

What did this study find?

Our study demonstrated an increasing incidence in dental injuries in patients aged 0-18 years and most dental injuries occurred during cycling, sports and playground-related activities.

Why does this study matter to clinicians?

Knowledge of dental injury patterns provides a framework for preventative education by health care providers by advocating for supervised play and cycling, and the use of appropriate protective equipment. 